\title{
Probing Gamma-Ray Burst VHE Emission with the Southern Wide-Field-of-View Gamma-Ray Observatory
}

\author{
Giovanni La Mura ${ }^{1, *,+}\left(\mathbb{D}\right.$, Ulisses Barres de Almeida ${ }^{2}\left(\mathbb{D}\right.$, Ruben Conceição ${ }^{1,3}$, Alessandro De Angelis ${ }^{4,5,6} \mathbb{D}^{\circ}$, \\ Francesco Longo ${ }^{7,8,9}$, , Mário Pimenta ${ }^{1,2}$, Bernardo Tomé ${ }^{1,3}{ }^{-1}$ and Davide Miceli ${ }^{10}$
}

1 Laboratório de Instrumentação e Física Experimental de Partículas (LIP), Av. Prof. Gama Pinto 2, 1649-003 Lisboa, Portugal; ruben@lip.pt (R.C.); pimenta@lip.pt (M.P.); bernardo@lip.pt (B.T.)

2 Brazilian Center for Physics Research (CBPF), Rua Dr. Xavier Sigaud 150, Rio de Janeiro 22290-180, Brazil; ulisses@cbpf.br

3 Instituto Superior Técnico (IST), Av. Rovisco Pais 1, 1049-001 Lisboa, Portugal

4 Dipartimento di Fisica e Astronomia-Università di Padova, Via Marzolo 8, 35131 Padova, Italy; alessandro.deangelis@unipd.it

5 Dipartimento di Scienze Matematiche, Informatiche e Fisiche-Università Degli Studi di Udine, Via Palladio 8, 33100 Udine, Italy

6 Istituto Nazionale di Fisica Nucleare sez. Padova (INFN), Via Marzolo 8, 35131 Padova, Italy

7 IFPU-Institute for Fundamental Physics of the Universe, Via Beirut 2, 34014 Trieste, Italy; francesco.longo@ts.infn.it

8 INFN, Sezione di Trieste, Via A. Valerio 2, 34100 Trieste, Italy

9 Dipartimento di Fisica, Università degli Studi di Trieste, Via A. Valerio 2, 34100 Trieste, Italy

10 Laboratoire d'Annecy de Physique des Particules (LAPP), CNRS-IN2P3, 9 Chemin de Bellevue-BP 110, 74941 Annecy, France; davide.miceli@lapp.in2p3.fr

check for updates

Citation: La Mura, G.; Barres de Almeida, U.; Conceição, R.; de Angelis, A.; Pimenta, M.; Tomé, B.; Miceli, D. Probing Gamma-Ray Burst VHE Emission with the Southern Wide-Field-of-View Gamma-Ray Observatory. Galaxies 2021, 9, 98. https: / / doi.org/10.3390/ galaxies 9040098

Academic Editor: Yi-Zhong Fan

Received: 29 September 2021 Accepted: 5 November 2021 Published: 8 November 2021

Publisher's Note: MDPI stays neutral with regard to jurisdictional claims in published maps and institutional affiliations.

Copyright: (c) 2021 by the authors. Licensee MDPI, Basel, Switzerland. This article is an open access article distributed under the terms and conditions of the Creative Commons Attribution (CC BY) license (https:// creativecommons.org/licenses/by/ $4.0 /)$.
* Correspondence: glamura@lip.pt or swgo_spokespersons@swgo.org

† Current Address: Laboratório de Instrumentação e Física Experimental de Partículas (LIP), Av. Prof. Gama Pinto 2, 1649-003 Lisboa, Portugal

Abstract: Recent observations have confirmed that Gamma-Ray Burst (GRB) afterglows produce Very High-Energy radiation (VHE, E > 100 GeV). This highly anticipated discovery opens new scenarios in the interpretation of GRBs and in their role as probes of Extragalactic Background Light (EBL) and Lorentz Invariance Violation (LIV). However, some fundamental questions about the actual nature of VHE emission in GRBs and its evolution during the burst are still unsolved. These questions will be difficult to address, even with future imaging Cherenkov telescopes, such as the Cherenkov Telescope Array (CTA). Here we investigate the prospects of gamma-ray sky monitoring with Extensive Air Showers arrays (EAS) to address these problems. We discuss the theoretical aspects connected with VHE radiation emission and the implications that its temporal evolution properties have on the interpretation of GRBs. By revisiting the high-energy properties of some Fermi-LAT detected GRBs, we estimate the typical fluxes expected in the VHE band and compare them with a range of foreseeable instrument performances, based on the Southern Wide Field-ofview Gamma-ray Observatory concept (SWGO). We focus our analysis on how different instrument capabilities affect the chances to explore the burst onset and early evolution in VHE, providing invaluable complementary information with respect to Cherenkov telescope observations. We show that under the assumption of conditions already observed in historical events, the next-generation ground monitoring detectors can actually contribute to answer several key questions.

Keywords: instrumentation—detectors; gamma rays: general; gamma-ray bursts: general

\section{Introduction}

The recent observation of Gamma-Ray Bursts (GRB) in the Very High-Energy domain (VHE, $E \geqslant 100 \mathrm{GeV}$ ) [1,2] marked an extraordinary milestone in our understanding of these outstandingly powerful transients. From the moment of their first identification as cosmological sources [3-5], it was immediately clear that their luminosity ranged up to 
values as high as $L \sim 10^{52} \mathrm{erg} \mathrm{s}^{-1}$, making them the brightest sources of electromagnetic radiation known in the Universe and requiring an extremely efficient energy production mechanism. At present it is thought that GRBs arise as the consequence of ultra-relativistic shocks in magnetized plasma jets, which are launched in the fast accretion process that follows the collapse of very massive stars $\left(M \geqslant 20 \mathrm{M}_{\odot}\right)$ or the merger of compact stellar remnants, such as neutron stars (NS) and black holes (BH), to form a magnetar or a new $\mathrm{BH}[6,7]$. The conversion of the huge amount of gravitational binding energy and thermal energy into kinetic and radiative power, within fractions of a second, triggers the emission of intense radiation, through mechanisms that naturally lead to the production of highenergy photons.

In general, a GRB is characterized by two emission regimes: an initial pulse of radiation, named prompt emission, most frequently observed in the energy range between $100 \mathrm{keV}$ and $1 \mathrm{MeV}$ and characterized by fast and strong variability [8], followed by a smoothly decaying afterglow phase, which can be detected from the radio and optical frequencies all the way up to energetic $\gamma$ rays. The prompt phase lasts only a few seconds and it can be used to distinguish between a class of short GRBs, where the prompt emission takes place for less than $2 \mathrm{~s}$, and one of long GRBs, whose prompt radiation is emitted for longer times [9-11]. These two classes can be fairly well interpreted by different types of source, with the long GRBs being more likely associated with a massive star core-collapse event $[12,13]$, while the short ones can be better reproduced in the compact binary merger scenario [7,14], as confirmed by the Multi-Messenger observation of GRB 170817A in connection with GW170817 [15-17].

Apart from the generally well-understood picture, the nature of GRBs and of their radiation mechanisms still poses many difficult questions. On one side, there are solid theoretical arguments that predict energetic radiation from relativistic magnetized plasmas. On the other hand, the explanation of the spectral and temporal properties is not totally consistent with simple leptonic scenarios. It is very likely that synchrotron emission should be the dominant radiation mechanism for GRBs, as argued, e.g., in [18-20]. However, the variability that is observed down to millisecond timescales in the prompt stage [21] implies compact emission regions, where the magnetic and radiative energy densities are so high that the radiative cooling should consequently be very fast and produce soft spectra [22]. This is inconsistent with the observational evidence [23,24]. Many different possibilities, invoking thermal components, re-acceleration mechanisms or non-leptonic scenarios e.g., [25-27], have been proposed to address this problem.

The observation of VHE photons has a crucial role in the identification of the radiation mechanisms at work. These photons can be produced as a consequence of shocks between relativistic blobs in the jet (internal shocks, probably dominant during the prompt stage), as well as between the jet and the external environment (external shocks, expected to occur in the afterglow). In addition, they require compact sources in relativistic motion, to escape the production site. VHE radiation can be observed at the ground, using either Imaging Atmospheric Cherenkov Telescopes (IACT), such as MAGIC [28] and H.E.S.S. [29], or Extensive Air Shower (EAS) particle detector arrays, such as HAWC [30] and LHAASO [31]. At present, IACTs have been able to firmly detect VHE emission in the afterglow of some powerful GRBs. It is very likely that the next-generation Cherenkov Telescope Array (CTA) [32] will further improve our ability to investigate the VHE signal of GRBs. However, due to their small field of view ( $\mathrm{FoV}$ ) and to the consequent requirement to be alerted and pointed towards the source, these instruments can only track GRBs with a certain delay after their actual onset.

Here we describe the scientific opportunities that can be explored by means of EAS arrays. Thanks to their large $\mathrm{FoV}$, which grants a continuous sky coverage of more than $1 \mathrm{sr}$, these instruments have a higher chance to probe the early phases of GRB emission, without the necessity of an external trigger. We discuss the issue taking into account the characteristics of an array concept based on Water Cherenkov Detectors (WCD) and investigated by the Southern Wide-field-of-view Gamma-ray Observatory collaboration 
(SWGO) [33]. Our work is structured as follows: in §2 we present the theoretical framework of GRB emission; in $\S 3$ we describe the known and the expected VHE properties of GRBs; in $\S 4$ we discuss the detection opportunities of an instrument such as SWGO; finally, in $\S 5$ we summarize our conclusions.

\section{Theoretical Framework}

Despite several decades of investigation, we do not yet have a complete theory for GRBs. What we know for sure is that the presence of high-energy $\gamma$ rays with a nonthermal spectrum implies emission from a highly relativistic source. This result stems from the well-known compactness problem [34,35]. A relatively simple argument can be used to illustrate the concept. A bright GRB has a time integrated energy flux, or fluence, of the order of $F \sim 10^{-7} \mathrm{erg} \mathrm{cm}^{-2}$, which is approximately related to the total emitted energy E by:

$$
E=4 \pi D_{L}^{2} F \approx 10^{50} \operatorname{erg}\left(\frac{D_{L}}{3000 \mathrm{Mpc}}\right)^{2}\left(\frac{F}{10^{-7} \mathrm{erg} \mathrm{cm}^{-2}}\right),
$$

where $D_{L}$ is the luminosity distance. The typical variability timescale is $\delta \mathrm{T} \approx 10^{-2} \mathrm{~s}$, implying an emitting region size limit $R \leqslant c \delta T \approx 3000 \mathrm{~km}$. As a result, the source would be characterized by an extremely high radiation density. High-energy photons can produce electron-positron pairs whenever the condition $\sqrt{E_{1} E_{2}} \geqslant 2 m_{e} c^{2}$ is met. Introducing a probability factor $f_{p}$ that accounts for the likelihood of the pair production mechanism, we obtain a pair production opacity of:

$$
\tau_{\gamma \gamma}=\frac{f_{p} \sigma_{T} F D_{L}^{2}}{R^{2} m_{e} c^{2}(1+z)} \approx \frac{10^{13} f_{p}}{1+z}\left(\frac{F}{10^{-7} \mathrm{erg} \mathrm{cm^{-2 }}}\right)\left(\frac{D_{L}}{3000 \mathrm{Mpc}}\right)^{2}\left(\frac{\delta T}{10^{-2} \mathrm{~s}}\right)^{-2}
$$

where $\sigma_{T}$ denotes the Thomson scattering cross-section. For typical GRB characteristics, the opacity predicted in Equation (2) is very large and should result in a thermal spectrum, in clear contradiction with observational evidence. If we allow the source to be a blob of plasma, approaching in a relativistic motion with bulk Lorentz factor $\Gamma$, at small angles with respect to the line of sight, and characterized by a power-law energy spectrum $N(\gamma) \propto \gamma^{-\alpha}$, the energy and the rate of arrival of the observed photons are both a factor $\Gamma$ higher than the corresponding values in the emission frame. Due to the Doppler effect on frequency, this implies that the photons that we observe at a given frequency are a factor of $\Gamma^{2 \alpha}$ denser than what would be seen in the emitting frame. The size of the emitting region is also affected by relativistic contraction, implying that $R_{e m} \leqslant \Gamma^{2} c \delta T$. Thus, Equation (2) should be corrected to:

$$
\tau_{\gamma \gamma} \approx \frac{10^{13} f_{p}}{(1+z) \Gamma^{(4+2 \alpha)}}\left(\frac{F}{10^{-7} \mathrm{erg} \mathrm{cm}^{-2}}\right)\left(\frac{D_{L}}{3000 \mathrm{Mpc}}\right)^{2}\left(\frac{\delta T}{10^{-2} \mathrm{~s}}\right)^{-2},
$$

that since $1<\alpha<3$ and $f_{p}<1$, predicts an optically thin regime when the condition $\Gamma \geqslant 10^{13 /(4+2 \alpha)} \approx 10^{2}$ is satisfied.

Similar order of magnitude considerations could be drawn to estimate the predicted spectrum. When reproducing GRB spectra starting from theoretical considerations, it is common use to introduce a set of free parameters containing some assumptions due to unknown properties of the detailed structure of the jet, the ongoing acceleration process and the shock micro-physics. In particular, an unknown fraction of the energy dissipated through the shock will go to the particle distribution and to the magnetic field. To account for this effect, two normalization parameters $\epsilon_{e}$ and $\epsilon_{B}$, expressing, respectively, the fractions of total energy carried by the particles and the magnetic field, are then introduced. When particles are accelerated in shock waves through the Fermi mechanism, we expect a resulting power-law energy distribution in the form of $N(\gamma) \propto \gamma^{-p}$ and magnetic fields that can be as large as $B \approx 10^{4} \mathrm{G}$ [36]. If the radiating species are electrons and positrons, 
the energy is quickly converted into radiation, through the emission of fast pulses of synchrotron and Compton scattered photons, with a spectral form similar to the one illustrated in Figure $1[37,38]$. As a result, we expect that GRBs should in principle be powerful sources of transient VHE emission, although the most energetic part of the spectrum is prone to pair production opacity on the Extragalactic Background Light (EBL) photons, which implies suppression of the most energetic radiation from sources located at large cosmological distances [39-41].

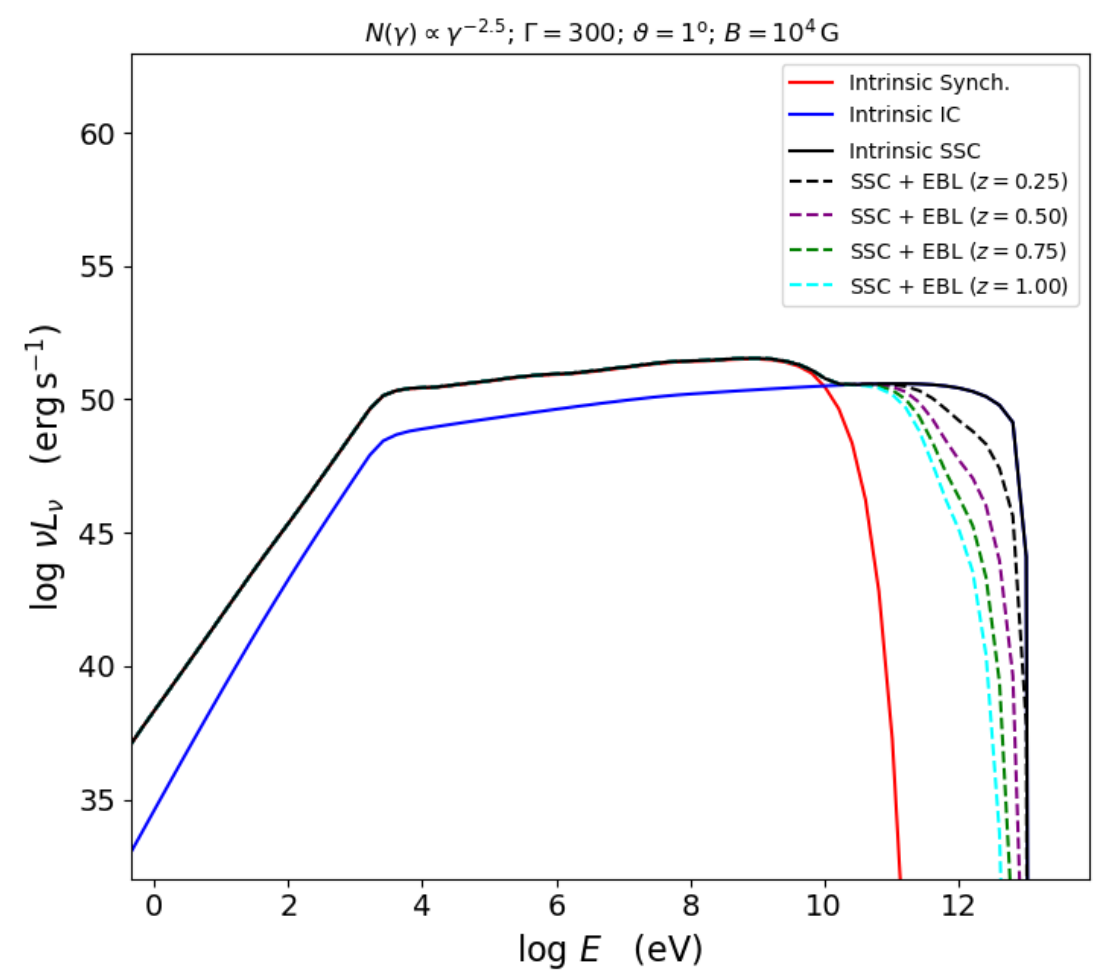

Figure 1. Spectral energy distribution expected for a relativistic blob of plasma, with bulk Lorentz factor $\Gamma=300$, moving with an inclination of $\vartheta=1^{\circ}$ from the line of sight and carrying a magnetic field $B=10^{4} \mathrm{G}$. The radiating particles are assumed to be electron-positron pairs in a power-law distribution $N(\gamma) \propto \gamma^{-2.5}$, with $10 \leqslant \gamma \leqslant 10^{6}$. The red line represents synchrotron emission, while the blue line shows the inverse Compton scattering contribution. The resulting spectrum is represented as a continuous black line. The dashed curves illustrate the effects of $\gamma \gamma$-opacity on the Universe background radiation for redshifts $0.25 \leqslant z \leqslant 1$, in steps of 0.25 .

Although the scenario depicted above can in principle justify the energies and the spectra that we see in GRBs, it is nonetheless prone to many important problems. The pulselike appearance of the prompt stage light curve is consistent with the presence of particle acceleration processes, followed by a rapid cooling. The typical burst duration, however, requires many acceleration events or an effective supply of energetic particles, to match the data. A critical aspect is the onset and the duration of the production of the most energetic photons [42]. If they are emitted as a continuation of the synchrotron spectrum, they should be highly correlated with the low energy radiation. On the contrary, the interaction of several emitting regions, or the presence of non-leptonic contributions, can lead to the prediction of delayed high-energy emission [43]. Although the currently available observations tend to favor a delayed detection of energetic photons, the existence of earlier VHE contributions is not ruled out and it represents a critical factor to discriminate between different possible scenarios. More accurate spectral models would need to take into account the evolution of the system and the probably important effects of non-homogeneity and orientation. We also must consider the possibility that the existence of very energetic photons (up to the $\mathrm{TeV}$ scale) in a presumably dense environment can lead to important 
photo-hadronic interactions and, therefore, to potentially much more elaborated spectral forms. This type of processes is actually expected to occur when the jet plasma collides with the external environment, therefore taking a major role in the afterglow emission. The true nature of GRBs in their initial stages, however, will only be clarified when precise spectral and temporal information on their most energetic emission at early times are obtained.

\section{VHE Properties of GRBs}

So far, the direct detection of VHE radiation from GRBs has only been possible for a limited number of cases, thanks to ground-based follow-up observations. The monitoring campaign carried out by the Fermi Large Area Telescope (Fermi-LAT) [44] led to the identification of a high-energy spectral component, coming in the form of power-law emission, which appears to be a common feature of bright GRBs and may even arise very early in the event [45]. Identifying the origin of this component and its relationship with the low energy portion of the spectrum has important implications on the interpretation of GRBs. If it arises as a high-energy extension of the synchrotron spectrum, we would expect a strong degree of correlation between different spectral bands, with direct implications on the energy of the radiating particles. If, on the contrary, it represents an independent contribution, its distribution among GRBs and its spectral characteristics may prove fundamental to understand its origin. The data collected so far, however, do not yet allow the drawing of a conclusive picture.

Due to its limited collecting area, the LAT cannot place strong constraints on the spectral features of short transients at $E \geqslant 100 \mathrm{GeV}$. In the assumption of a composite synchrotron and inverse Compton spectrum, the upper limits placed above $\sim 30 \mathrm{GeV}$ for the VHE detected GRB 190114C represented an invaluable reference to estimate the transition between the two regimes, though the possibility of alternative interpretations and the limited temporal information still leave room for open questions. In any case, the results of Fermi-LAT observations can be used as a starting point to estimate the possible extension of the GRB properties to the VHE domain and therefore evaluate their detection possibility with other instruments.

Assuming, for the sake of simplicity, that the temporal evolution of the spectrum is only limited to a scaling factor, without relevant spectral changes, we can express the high-energy spectrum of a GRB as a function of energy in the form of:

$$
\frac{\mathrm{d} N(t)}{\mathrm{d} E}=N_{0}(t)\left(\frac{E}{E_{0}}\right)^{-\alpha} \exp [-\tau(E, z)] \quad\left[\text { photons } \mathrm{cm}^{-2} \mathrm{~s}^{-1} \mathrm{GeV}^{-1}\right],
$$

where $N_{0}(t)$ is the flux of photons per unit energy observed at time $t$ and pivot energy $E_{0}$, $\alpha$ is the spectral index, which is often within the range $1.5 \leqslant \alpha \leqslant 3$, with an average value close to 2 , and $\tau(E, z)$ is the opacity due to pair production on EBL, given as a function of energy and redshift. The temporal evolution of the flux is typically well represented by a power-law, or a broken power-law, which can be written as:

$$
N_{0}(t)= \begin{cases}N_{\text {peak }}\left(\frac{t-T_{0}}{T_{\text {peak }}-T_{0}}\right) & \text { for } \quad T_{0} \leqslant t<T_{\text {peak }} \\ N_{\text {peak }}\left(\frac{t}{T_{\text {peak }}}\right)^{-\gamma} & \text { for } t \geqslant T_{\text {peak }}\end{cases}
$$

where we denoted with $T_{0}$ the trigger time, with $T_{\text {peak }}$ the time taken to achieve peak emission, with $N_{\text {peak }}$ the maximum flux, and $\gamma$ the temporal evolution index, which is often found to be $1 \leqslant \gamma \leqslant 2$.

Using the second catalog of Fermi-LAT detected GRBs (2FLGC) [45], which provides measurements of the observed photon fluxes in the energy range between $100 \mathrm{MeV}$ and $10 \mathrm{GeV}$, together with information on the spectral index and on the light-curve shape, for a sample of GRBs observed during 10 years of regular monitoring operations, we are able to apply Equation (4), with the inclusion of Equation (5), to estimate the expected 
high-energy fluxes as a function of time, as illustrated for instance in Figure 2. In principle, we can extend this type of spectra to the VHE domain and, thus, obtain an estimate for the expected fluxes. In practice, this operation is not directly possible, due to the lack of a redshift measurement for most of the LAT detected GRBs, which implies an unknown EBL opacity in Equation (4). Although the effects of EBL are generally negligible for the observed LAT band, they become quickly very important at higher energies, with a typical EBL opacity horizon set by $\tau=1$ for $z \approx 1$ already at $E=100 \mathrm{GeV}$ [41]. For this reason, we combined the spectral and temporal fits, which we obtained from the LAT data, with a set of simulations, aiming at estimating the effects of EBL opacity on the VHE extension of the GRBs that resulted in the observed LAT fluxes.

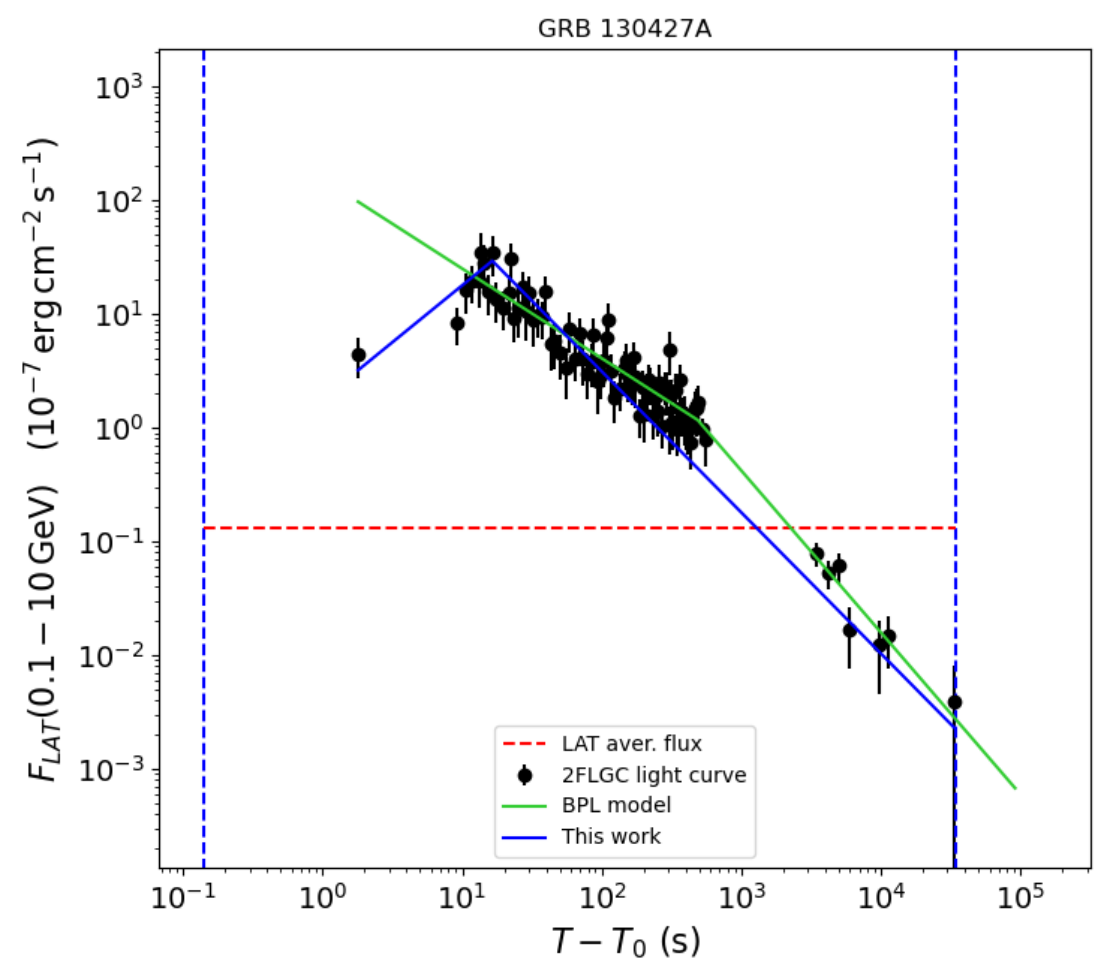

Figure 2. Comparison between the Fermi-LAT light curve of GRB 130427A and the model based on Equations (4) and (5). The vertical blue dashed lines mark the temporal window of the LAT signal, the red horizontal line is the average energy flux collected during the emission, the green continuous line is the 2FLGC broken power-law fit to the data, while the blue continuous line is a model using the light curve of Equation (5).

The approach that we adopted in our simulations was to extract the fluences of all the LAT detected GRBs, reported in 2FLGC, and to assign a set of 1000 random redshift values to each GRB without an available redshift measurement. The result of this process is the production of 1000 random GRB redshift distributions, corresponding to an equal number of random luminosity distributions, all of which yield the observed 2FLGC population. As shown in Figure 3, the simulation set provides a distribution of GRB luminosities which is in good agreement with the one followed by the 2FLGC GRBs with a measured redshift. The combination of all the different simulations, therefore, can be used to estimate the likelihood that a GRB with measured spectral and temporal characteristics is associated with a specific redshift range (see [46] for more details on the simulation process). 


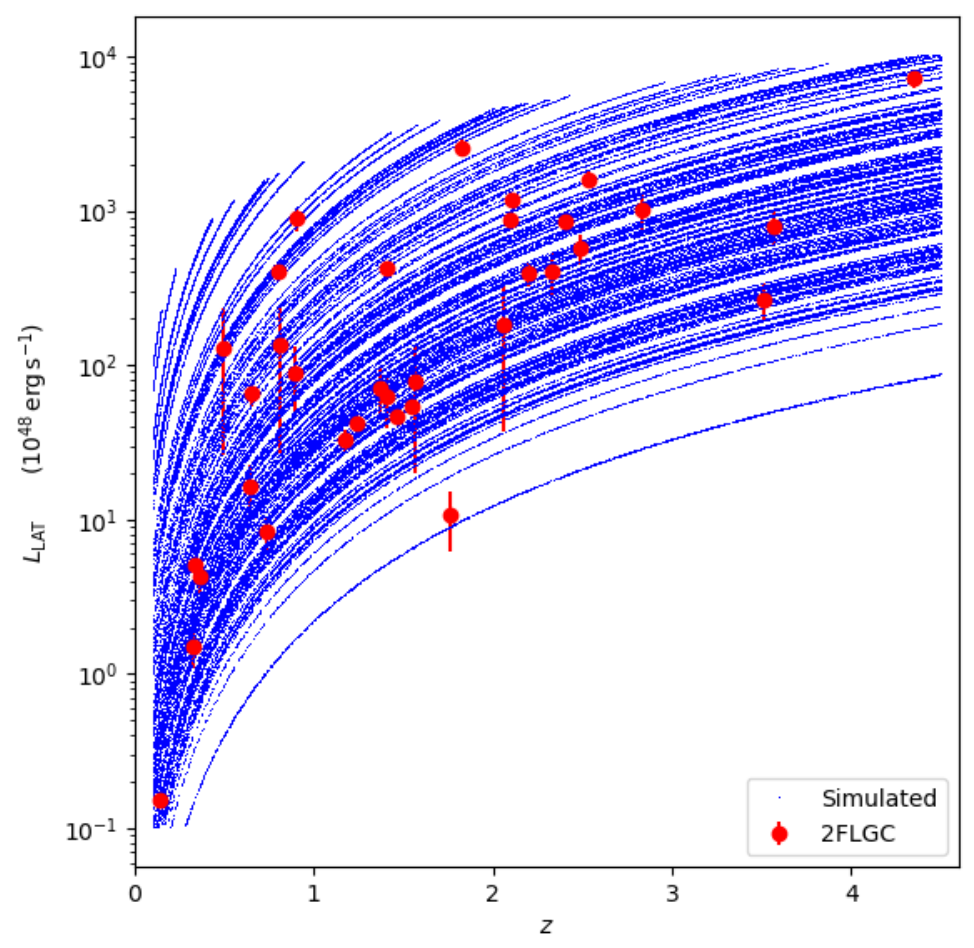

Figure 3. Comparison between the luminosity estimated in the LAT band $0.1 \mathrm{GeV} \leqslant E \leqslant 10 \mathrm{GeV}$ for the 2FLGC GRBs with a redshift measurement (large red dots) and the luminosity of the 2FLGC GRBs with 1000 randomly distributed redshifts (small blue points).

\section{Monitoring GRBs with SWGO}

Both theoretical and observational arguments suggest that VHE emission is an important property of GRBs. This is a well verified feature in the afterglow of some bright events. Evidence for the existence of energetic photons in the prompt phase is much harder to obtain, although there are some Fermi-LAT detected bursts that hint in this direction. Solving questions such as the occurrence rate of energetic spectral components in GRB afterglows, the existence of fast VHE pulses associated with the prompt stage, and assess the delay in the onset of VHE components is a problem that requires an extensive monitoring campaign, possibly covering a large sample of GRBs with an instrument characterized by a large collecting area. At present, several experiments are available or are being constructed to provide VHE spectral coverage with different sensitivities and resolution.

The SWGO collaboration is currently investigating the design of a new WCD arraybased EAS observatory, to be constructed in the Southern Hemisphere [47]. The target performance domain is summarized in Figure 4, together with the sensitivities achieved by other instruments and with a comparison of the expected limiting fluxes with the ones emitted by GRBs that were detected in the HE and VHE domains. The role of such a new instrument will be to provide constant scanning of a wide FoV in the Southern sky, thus complementing the FoV covered by Northern facilities such as HAWC and LHAASO and providing a triggering and alert system for CTA. Adopting a compact array concept with a collecting area of $80,000 \mathrm{~m}^{2}$, located in a high-altitude site $(\geqslant 4400 \mathrm{~m}$ a.s.l.), this type of instrument has the possibility to probe the flux range that we expect to be characteristic of GRB emission, with a transient localization accuracy $\alpha_{68} \leqslant 1^{\circ}$ below $1 \mathrm{TeV}$, although the possibility to detect different types of events depends critically on the overall instrument performance and on its ability to probe the lower part of the spectral range, where the effects of EBL opacity are less severe and a larger volume of the Universe is potentially accessible. 

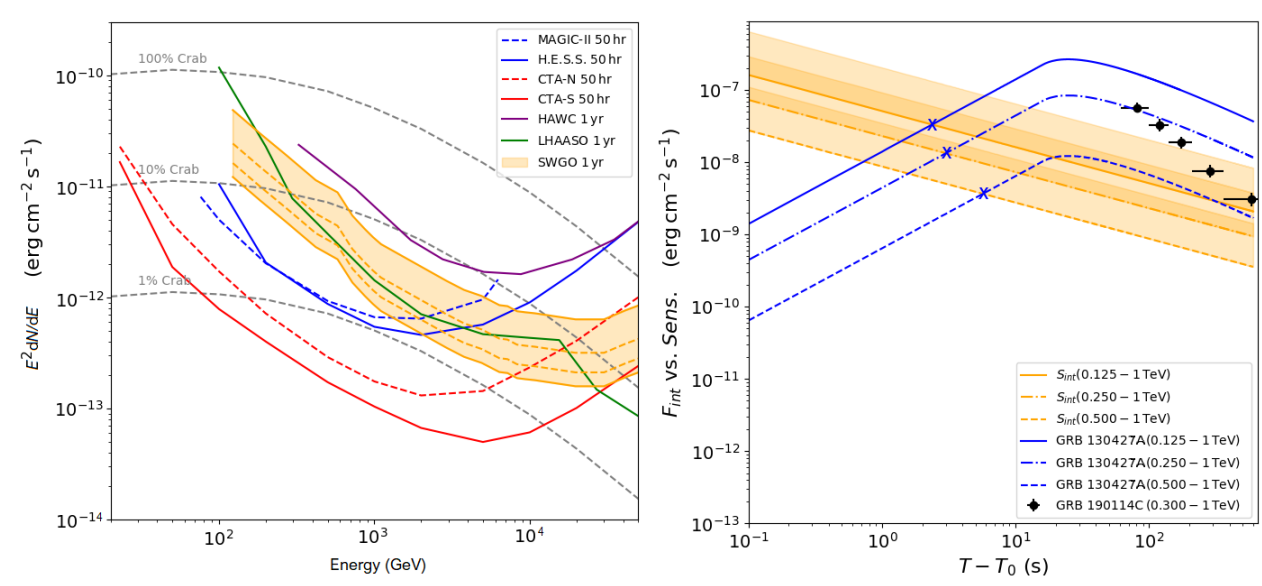

Figure 4. (Left panel) Differential sensitivity to the flux of a point-like source located at zenith distance $\vartheta=20^{\circ}$ for SWGO, HAWC and LHAASO (computed for 1 year of data taking) and for MAGIC, H.E.S.S. and CTA (computed for $50 \mathrm{~h}$ of exposure time). Different fractions of the Crab Nebula flux are also shown for comparison. (Right panel) Expected detection times for a GRB with the temporal and spectral characteristics of GRB 130427A, located at redshift $z=0.34$, for different fractions of the optimal SWGO performance, taken integrating the spectrum above thresholds of $E=125 \mathrm{GeV}, 250 \mathrm{GeV}$ and $500 \mathrm{GeV}$. For each case, the blue crosses mark the time required to accumulate an integrated flux above the corresponding detection threshold. The black points with error bars denote the temporal evolution of the VHE flux detected by MAGIC for $E \geqslant 300 \mathrm{GeV}$ from GRB 190114C [1].

To test the potential role of SWGO as a monitoring and alert system, we took the sample of GRBs with simulated redshifts, discussed in $\S 3$, and we calculated the expected VHE fluxes, integrating Equation (4), with the inclusion of Equation (5), in time and in energy, using the spectral and the temporal characteristics extracted from 2FLGC and applying the $\gamma \gamma$ absorption effects predicted by an EBL opacity model [39]. The results of these calculations are summarized in Figure 5 for different possible performances of the experiment. The plots shown in Figure 5 represent the number of simulated redshift distributions that result in the GRB detections reported on the $x$-axes out of a total of 1000 simulations. Although we observe that all the explored configurations have some degree of detection chances, we can easily verify that an instrument performing at the optimal sensitivity, down to a low energy threshold of $E_{\text {low }}=125 \mathrm{GeV}$, has a predicted ability to detect significantly more than 10 GRBs in 10 years in approximately $75 \%$ of the simulated scenarios, provided that they occur within a zenith distance of $\vartheta \leqslant 20^{\circ}$. Adopting lower performance solutions, or using a spectral window with a higher limiting threshold, such as $250 \mathrm{GeV}$ or $500 \mathrm{GeV}$, leads to lower detection chances, due to the loss of many relatively low-flux events and to a more severe effect of EBL opacity, which limits the visible horizon to a smaller volume of the Universe. Incidentally, these calculations approximately correspond to what we would expect at larger zenith distances and, therefore, within increasing fractions of the available FoV, from an optimally performing solution. As a result, even if the FoV covered by a ground-based instrument below $1 \mathrm{TeV}$ is smaller than the one that was available in the case of the Fermi-LAT observations, by taking into account the estimated distribution of expected GRB fluxes, we can conclude that the possibility to detect more than 1 event per year is a reasonable expectation. Due to the characteristic shapes of the tested light-curves, which are generally dominated by the peak flux, a fraction of approximately $(78 \pm 16) \%$ of these detections is estimated to occur within the first $10 \mathrm{~s}$ of the event, resulting in promisingly good chances to investigate the elusive properties of the prompt emission. 

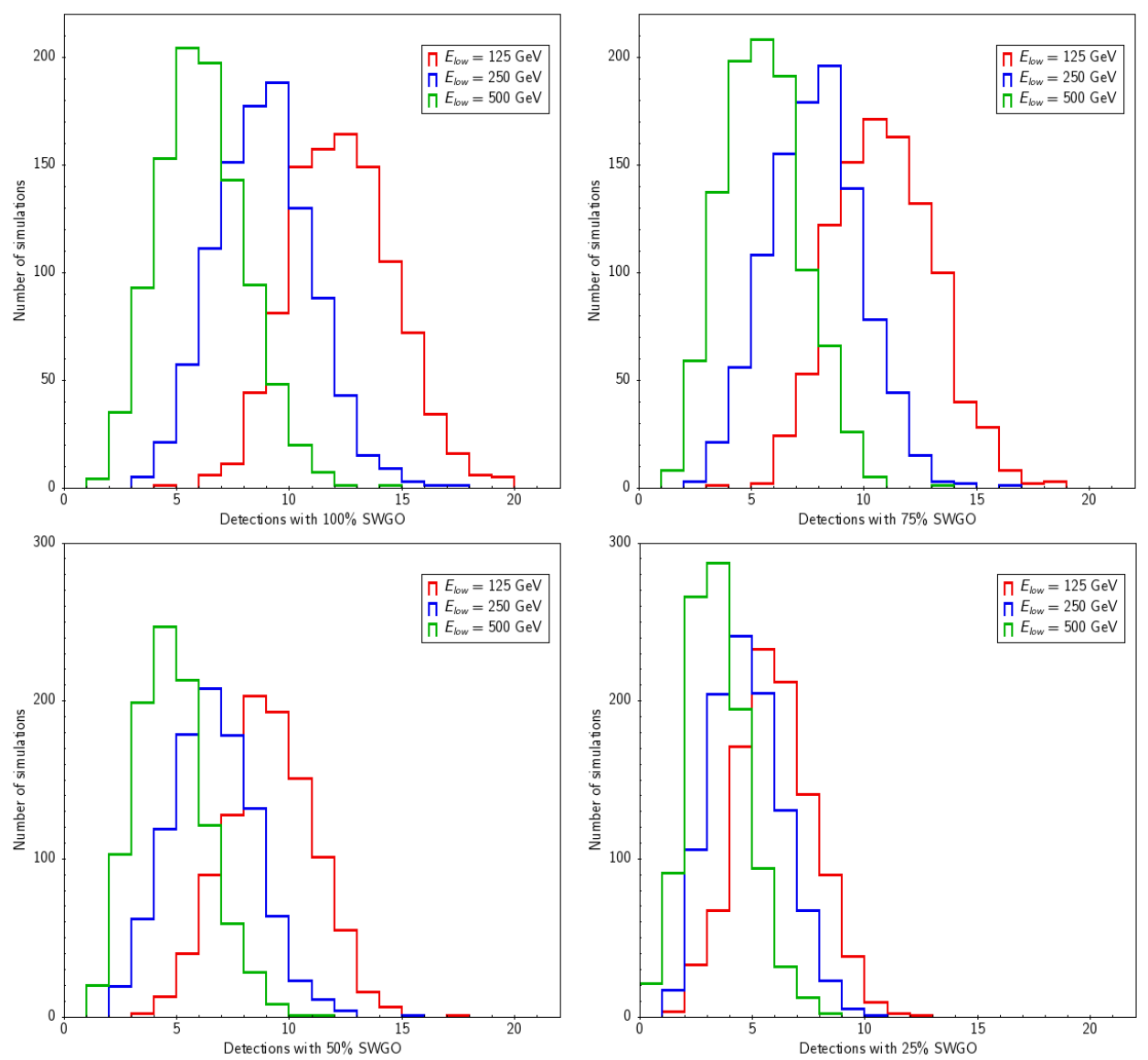

Figure 5. From upper left to lower right: histograms of the number of expected detections of GRBs extracted from 2FLGC and associated with 1000 random redshift values, computed for flux integrations above a low energy threshold of $E_{\text {low }}=125 \mathrm{GeV}$ (red histograms), $250 \mathrm{GeV}$ (blue histograms) and $500 \mathrm{GeV}$ (green histograms), with an overall performance reaching up to $100 \%, 75 \%$, $50 \%$ and $25 \%$ of the optimal SWGO sensitivity and zenith distance up to $\vartheta=20^{\circ}$. The optimal SWGO design is expected to be able to detect more than 10 GRBs in an observing period of 10 years in more than 750 simulations out of 1000 . Reductions of performance or higher energy thresholds reflect in gradually lower expected detection chances.

\section{Conclusions}

Investigating the VHE properties of GRB will have fundamental implications in our understanding of these extremely powerful events. The existence of VHE radiation components, particularly if associated with the prompt stage, represents a fundamental piece of information to model the physics of the radiating environment, thanks to the strong implications that VHE spectral and temporal properties have on the radiating species. The degree of correlation, or the occurrence of delays of the energetic components with respect to lower energy emission, depend on the particle energy distribution and on the interactions within the emitting regions, or between these regions and the environment. Testing the distribution of these properties among long and short GRBs will reduce the ambiguity implied by the partial overlap of these classes and further characterize the jets produced in the two cases. In addition, the possibility to verify whether the early VHE emission is dominated by smooth temporal evolution or irregular variability will improve our understanding of the transition between the prompt stage and the afterglow emission, providing invaluable information on the close GRB environment.

In our investigation, we used the HE data from GRBs detected during the first 10 years of Fermi-LAT observations, to infer the expected properties of GRBs in the VHE domain. Using the spectral and timing information of the LAT detected events, to estimate the expected fluxes, with the aid of ancillary simulations to derive fiducial redshift distributions 
for most of the sources with unknown $z$, we extrapolated the predicted spectra up to the $\mathrm{TeV}$ scale, taking into account the effects of EBL opacity. We then calculated the detection prospects of the resulting GRB distributions for different performances of the experiment, based on estimates of the SWGO potential. We found that a new monitoring facility, with the characteristics investigated by the SWGO collaboration, could effectively monitor VHE emission from GRBs, providing localization information at the level of a few square degrees and reaching timescales of less than $10 \mathrm{~s}$ for the brightest events. This is a time domain that is very hard to explore with IACT facilities, despite being critical to distinguish whether the high-energy component is a spectral extension, characteristic of powerful bursts, or the result of an additional process, dominated by some external contribution. With the ability to trigger on VHE transient signals, the localization regions of Multi-Messenger source candidates will further improve and the efficiency in the execution of follow-up campaigns will subsequently increase.

Author Contributions: Conceptualization, U.B.d.A. and F.L.; methodology, G.L.M.; software, G.L.M., R.C. and B.T.; validation, F.L.; investigation, M.P. and A.d.A.; writing-original draft preparation, G.L.M.; funding acquisition, M.P. and U.B.d.A.; modeling, D.M. and G.L.M.; All authors have read and agreed to the published version of the manuscript.

Funding: This research was funded by Fundação para a Ciência e Tecnologia, under project PTDC/FIS-PAR/4300/2020 and grant DL57/2016/cP1330/cT0002, by CNPq Productivity Research, Grant no. 311997/2019-8, by Serrapilheira Institute Grant number Serra-1812-26906, and by FAPERJ Young Scientist Fellowship no. E-26/202.818/2019.

Institutional Review Board Statement: Not applicable.

Informed Consent Statement: Not applicable.

Data Availability Statement: There are no new data associated with this article. The data used to estimate the GRB HE properties are provided by the Fermi-LAT collaboration and publicly available online at the HEASARC website https:/ / heasarc.gsfc.nasa.gov/W3Browse/fermi/fermilgrb.html (accessed on 20 September 2021).

Acknowledgments: The authors gratefully thank Edna Ruiz-Velasco and Brian Reville for discussion and comments leading to the improvement of the manuscript. The SWGO Collaboration acknowledges the support from the agencies and organizations listed here: https:/ /www.swgo.org/ SWGOWiki/doku.php?id=acknowledgements (accessed on 20 September 2021). Part of this work is based on public data provided by the Fermi-LAT Collaboration. The Fermi-LAT Collaboration acknowledges generous ongoing support from several agencies and institutes that have supported both the development and the operation of the LAT as well as scientific data analysis. These include the National Aeronautics and Space Administration and the Department of Energy in the United States, the Commissariat à l'Energie Atomique and the Centre National de la Recherche Scientifique/Institut National de Physique Nucléaire et de Physique des Particules in France, the Agenzia Spaziale Italiana and the Istituto Nazionale di Fisica Nucleare in Italy, the Ministry of Education, Culture, Sports, Science and Technology (MEXT), High-Energy Accelerator Research Organization (KEK) and Japan Aerospace Exploration Agency (JAXA) in Japan, and the K. A. Wallenberg Foundation, the Swedish Research Council and the Swedish National Space Agency in Sweden. Additional support for science analysis during the operations phase is gratefully acknowledged from the Istituto Nazionale di Astrofisica in Italy and the Centre National d'Études Spatiales in France. This work performed in part under DOE Contract DEAC02-76SF00515.

Conflicts of Interest: The authors declare no conflict of interest. 


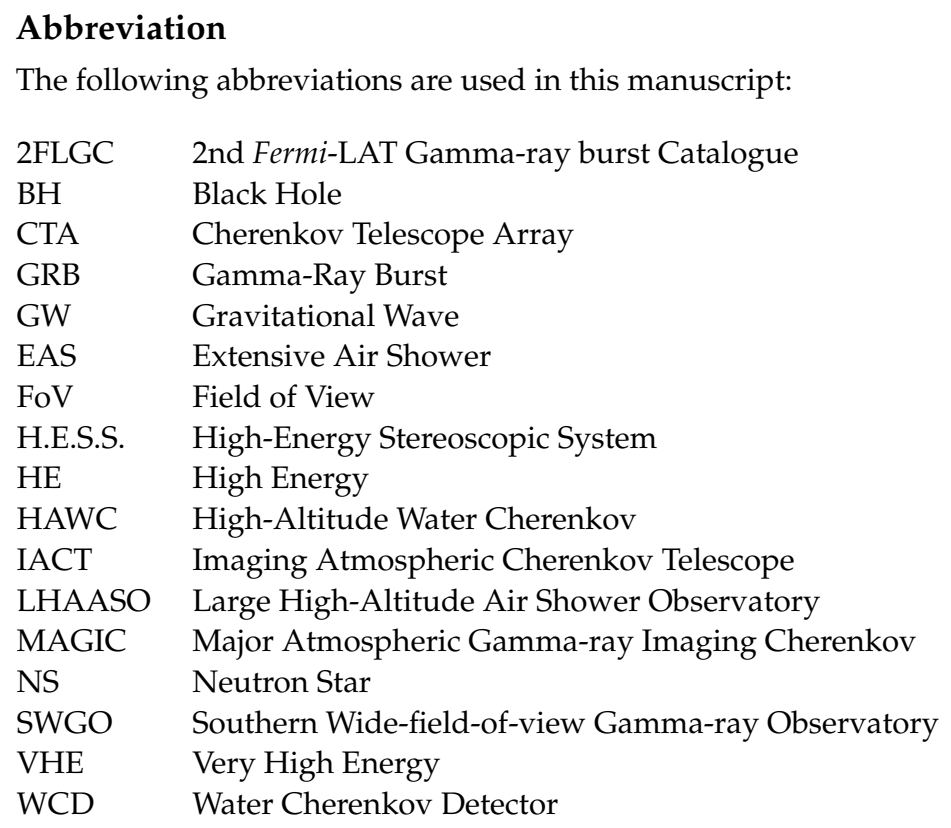

\section{References}

1. MAGIC Collaboration. Teraelectronvolt emission from the $\gamma$-ray burst GRB 190114C. Nature 2019, 575, 455-458. [CrossRef] [PubMed]

2. Abdalla, H.; Adam, R.; Aharonian, F.; Ait Benkhali, F.; Angüner, E.O.; Arakawa, M.; Arcaro, C.; Armand, C.; Ashkar, H.; Backes, M.; et al. A very-high-energy component deep in the $\gamma$-ray burst afterglow. Nature 2019, 575, 464-467. [CrossRef] [PubMed]

3. Fishman, G.J.; Meegan, C.A. Gamma-Ray Bursts. Annu. Rev. Astron. Astrophys. 1995, 33, 415-458. [CrossRef]

4. Metzger, M.R.; Djorgovski, S.G.; Kulkarni, S.R.; Steidel, C.C.; Adelberger, K.L.; Frail, D.A.; Costa, E.; Frontera, F. Spectral constraints on the redshift of the optical counterpart to the $\gamma$-ray burst of 8 May 1997. Nature 1997, 387, 878-880. [CrossRef]

5. Kulkarni, S.R.; Djorgovski, S.G.; Ramaprakash, A.N.; Goodrich, R.; Bloom, J.S.; Adelberger, K.L.; Kundic, T.; Lubin, L.; Frail, D.A.; Frontera, F.; et al. Identification of a host galaxy at redshift $\mathrm{z}=3.42$ for the $\gamma$-ray burst of 14 December 1997. Nature 1998, 393, 35-39. [CrossRef]

6. Woosley, S.E. Gamma-Ray Bursts from Stellar Mass Accretion Disks around Black Holes. Astrophys. J. 1993, 405, 273. [CrossRef]

7. Eichler, D.; Livio, M.; Piran, T.; Schramm, D.N. Nucleosynthesis, neutrino bursts and $\gamma$-rays from coalescing neutron stars. Nature 1989, 340, 126-128. [CrossRef]

8. Nakar, E.; Piran, T. Temporal properties of short gamma-ray bursts. Mon. Not. R. Astron. Soc. 2002, 330, 920-926. [CrossRef]

9. Mazets, E.P.; Golenetskii, S.V.; Ilinskii, V.N.; Panov, V.N.; Aptekar, R.L.; Gurian, I.A.; Proskura, M.P.; Sokolov, I.A.; Sokolova, Z.I.; Kharitonova, T.V. Catalog of cosmic gamma-ray bursts from the KONUS experiment data. Astrophys. Space Sci. 1981, 80, 3-83. [CrossRef]

10. Norris, J.P.; Cline, T.L.; Desai, U.D.; Teegarden, B.J. Frequency of fast, narrow $\gamma$-ray bursts. Nature 1984, 308, 434-435. [CrossRef]

11. Kouveliotou, C.; Meegan, C.A.; Fishman, G.J.; Bhat, N.P.; Briggs, M.S.; Koshut, T.M.; Paciesas, W.S.; Pendleton, G.N. Identification of Two Classes of Gamma-Ray Bursts. Astrophys. J. Lett. 1993, 413, L101. [CrossRef]

12. Galama, T.J.; Vreeswijk, P.M.; van Paradijs, J.; Kouveliotou, C.; Augusteijn, T.; Böhnhardt, H.; Brewer, J.P.; Doublier, V.; Gonzalez, J.-F.; Leibundgut, B.; et al. An unusual supernova in the error box of the $\gamma$-ray burst of 25 April $1998 . \quad$ Nature 1998, 395, 670-672. [CrossRef]

13. Bloom, J.S.; Kulkarni, S.R.; Djorgovski, S.G.; Eichelberger, A.C.; Côté, P.; Blakeslee, J.P.; Odewahn, S.C.; Harrison, F.A.; Frail, D.A.; Filippenko, A.V.; et al. The unusual afterglow of the $\gamma$-ray burst of 26 March 1998 as evidence for a supernova connection. Nature 1999, 401, 453-456. [CrossRef]

14. Li, L.X.; Paczyński, B. Transient Events from Neutron Star Mergers. Astrophys. J. Lett. 1998, 507, L59-L62. [CrossRef]

15. Abbott, B.P.; Abbott, R.; Abbott, T.D.; Acernese, F.; Adams, C.; Adams, T.; Addesso, P.; Adhikari, R.X.; Adya, V.B.; Affeldt, C.; et al. Multi-messenger Observations of a Binary Neutron Star Merger. Astrophys. J. Lett. 2017, 848, L12. [CrossRef]

16. Abbott, B.P.; Abbott, R.; Abbott, T.D.; Acernese, F.; Ackley, K.; Adams, C.; Adams, T.; Addesso, P.; Adhikari, R.X.; Adya, V.B.; et al. Gravitational Waves and Gamma-Rays from a Binary Neutron Star Merger: GW170817 and GRB 170817A. Astrophys. J. Lett. 2017, 848, L13. [CrossRef]

17. Cowperthwaite, P.S.; Berger, E.; Villar, V.A.; Metzger, B.D.; Nicholl, M.; Chornock, R.; Blanchard, P.K.; Fong, W.; Margutti, R.; Soares-Santos, M.; et al. The Electromagnetic Counterpart of the Binary Neutron Star Merger LIGO/Virgo GW170817. II. UV, Optical, and Near-infrared Light Curves and Comparison to Kilonova Models. Astrophys. J. Lett. 2017, 848, L17. [CrossRef]

18. Katz, J.I. Delayed Hard Photons from Gamma-Ray Bursts. Astrophys. J. Lett. 1994, 432, L27. [CrossRef] 
19. Rees, M.J.; Meszaros, P. Unsteady Outflow Models for Cosmological Gamma-Ray Bursts. Astrophys. J. Lett. 1994, 430 , L93. [CrossRef]

20. Tavani, M. Shock Emission Model for Gamma-Ray Bursts. Phys. Rev. Lett. 1996, 76, 3478-3481. [CrossRef]

21. Walker, K.C.; Schaefer, B.E.; Fenimore, E.E. Gamma-Ray Bursts Have Millisecond Variability. Astrophys. J. 2000, 537, $264-269$. [CrossRef]

22. Ghisellini, G.; Celotti, A. Quasi-thermal Comptonization and Gamma-Ray Bursts. Astrophys. J. Lett. 1999, 511, L93-L96. [CrossRef]

23. Band, D.; Matteson, J.; Ford, L.; Schaefer, B.; Palmer, D.; Teegarden, B.; Cline, T.; Briggs, M.; Paciesas, W.; Pendleton, G.; et al. BATSE Observations of Gamma-Ray Burst Spectra. I. Spectral Diversity. Astrophys. J. 1993, 413, 281. [CrossRef]

24. Preece, R.D.; Briggs, M.S.; Mallozzi, R.S.; Pendleton, G.N.; Paciesas, W.S.; Band, D.L. The Synchrotron Shock Model Confronts a "Line of Death" in the BATSE Gamma-Ray Burst Data. Astrophys. J. Lett. 1998, 506, L23-L26. [CrossRef]

25. Vietri, M. GeV Photons from Ultrahigh Energy Cosmic Rays Accelerated in Gamma Ray Bursts. Phys. Rev. Lett. 1997, 78, 4328-4331. [CrossRef]

26. Ghirlanda, G.; Pescalli, A.; Ghisellini, G. Photospheric emission throughout GRB 100507 detected by Fermi. Mon. Not. R. Astron. Soc. 2013, 432, 3237-3244. [CrossRef]

27. Burgess, J.M.; Preece, R.D.; Ryde, F.; Veres, P.; Mészáros, P.; Connaughton, V.; Briggs, M.; Pe'er, A.; Iyyani, S.; Goldstein, A.; et al. An Observed Correlation between Thermal and Non-thermal Emission in Gamma-Ray Bursts. Astrophys. J. Lett. 2014, 784, L43. [CrossRef]

28. Aleksić, J.; Ansoldi, S.; Antonelli, L.A.; Antoranz, P.; Babic, A.; Bangale, P.; Barceló. M.; Barrio, J.A.; Becerra González, J.; Bednarek, W. The major upgrade of the MAGIC telescopes, Part I: The hardware improvements and the commissioning of the system. Astropart. Phys. 2016, 72, 61-75. [CrossRef]

29. Aharonian, F.; Akhperjanian, A.G.; Bazer-Bachi, A.R.; Beilicke, M.; Benbow, W.; Berge, D.; Bernlöhr, K.; Boisson, C.; Bolz, O.; Borrel, V.; et al. Observations of the Crab nebula with HESS. Astron. Astrophys. 2006, 457, 899-915. [CrossRef]

30. DeYoung, T. The HAWC observatory. Nucl. Instruments Methods Phys. Res. A 2012, 692, 72-76. [CrossRef]

31. Zhen, C.; Ming-Jun, C.; Song-Zhan, C.; Hong-Bo, H.; Cheng, L.; Ye, L.; Ling-ling, M.; Xin-hua, M.; Xiang-dong, S.; Han-rong, W.; et al. Introduction to Large High Altitude Air Shower Observatory (LHAASO). Chin. Astron. Astrophys. 2019, 43, 457-478. [CrossRef]

32. CTA Consortium. Science with the Cherenkov Telescope Array; World Scientific Publishing Co.: Singapore, 2019. [CrossRef]

33. Barres de Almeida, U.; Giacinti, G.; Longo, F. Benchmarking the Science for the Southern Wide-Field Gamma-ray Observatory (SWGO). In Proceedings of the 37th International Cosmic Ray Conference (ICRC2021), Berlin, Germany, 12-23 July 2021; Volume 395, p. 10.

34. Ruderman, M. Theories of gamma -ray bursts. In Seventh Texas Symposium on Relativistic Astrophysics; Bergman, P.G., Fenyves, E.J., Motz, L., Eds.; John Wiley \& Sons, Ltd.: Hoboken, NJ, USA, 1975; Volume 262, pp. 164-180. [CrossRef]

35. Schmidt, W.K.H. Distance limit for a class of model $\gamma$-ray burst sources. Nature 1978, 271, 525-527. [CrossRef]

36. Piran, T. Gamma-ray bursts and the fireball model. Phys. Rep. 1999, 314, 575-667. [CrossRef]

37. Rybicki, G.B.; Lightman, A.P. Radiative Processes in Astrophysics; John Wiley \& Sons, Ltd.: Hoboken, NJ, USA, 1986. [CrossRef]

38. Xue, R.R.; Tam, P.H.; Wagner, S.J.; Behera, B.; Fan, Y.Z.; Wei, D.M. Very High Energy $\gamma$-Ray Afterglow Emission of Nearby Gamma-Ray Bursts. Astrophys. J. 2009, 703, 60. [CrossRef]

39. Dominguez, A.; Primack, J.R.; Rosario, D.J.; Prada, F.; Gilmore, R.C.; Faber, S.M.; Koo, D.C.; Somerville, R.S.; Pérez-Torres, M.A.; Pérez-González, P.; et al. Extragalactic background light inferred from AEGIS galaxy-SED-type fractions. Mon. Not. R. Astron. Soc. 2011, 410, 2556-2578. [CrossRef]

40. Franceschini, A.; Foffano, L.; Prandini, E.; Tavecchio, F. Very high-energy constraints on the infrared extragalactic background light. Astron. Astrophys. 2019, 629, A2. [CrossRef]

41. Saldana-Lopez, A.; Domínguez, A.; Pérez-González, P.G.; Finke, J.; Ajello, M.; Primack, J.R.; Paliya, V.S.; Desai, A. An observational determination of the evolving extragalactic background light from the multiwavelength HST/CANDELS survey in the Fermi and CTA era. Mon. Not. R. Astron. Soc. 2021. [CrossRef]

42. Fan, Y.Z.; Piran, T. High-energy $\gamma$-ray emission from gamma-ray bursts-Before GLAST. Front. Phys. China 2008, 3, 306. [CrossRef]

43. Asano, K.; Mészáros, P. Delayed Onset of High-energy Emissions in Leptonic and Hadronic Models of Gamma-Ray Bursts. Astrophys. J. 2012, 757, 115. [CrossRef]

44. Atwood, W.B.; Abdo, A.A.; Ackermann, M.; Althouse, W.; Anderson, B.; Axelsson, M.; Baldini, L.; Ballet, J.; Band, D.L.; Barbiellini, G.; et al. The Large Area Telescope on the Fermi Gamma-Ray Space Telescope Mission. Astrophys. J. 2009, 697, 1071-1102. [CrossRef]

45. Ajello, M.; Arimoto, M.; Axelsson, M.; Baldini, L.; Barbiellini, G.; Bastieri, D.; Bellazzini, R.; Bhat, P.N.; Bissaldi, E.; Blandford, R.D.; et al.; A Decade of Gamma-Ray Bursts Observed by Fermi-LAT: The Second GRB Catalog. Astrophys. J. 2019, 878, 52. [CrossRef] 
46. La Mura, G.; Barres de Almeida, U.; Conceição, R.; De Angelis, A.; Longo, F.; Pimenta, M.; Prandini, E.; Ruiz-Velasco, E.; Tomé, B. Gamma-ray burst detection prospects for next generation ground-based VHE facilities. Mon. Not. R. Astron. Soc. 2021, 508, 671-679. [CrossRef]

47. Schoorlemmer, H.; Conceição, R.; Smith, A.J. Simulating the performance of the Southern Wide-view Gamma-ray Observatory. In Proceedings of the 37th International Cosmic Ray Conference (ICRC2021). In Proceedings of the 37th International Cosmic Ray Conference (ICRC2021), Berlin, Germany, 12-23 July 2021; Volume 395, p. 9. 